\title{
CLINICAL ASPECTS OF COMPARATIVE SPECTROPHOTOMETRIC ANALYSIS IN THE SELECTION OF THE COLOUR OF ACRYLIC TEETH IN REMOVABLE DENTURES
}

\author{
Piotr Napadłek, Zdzisław A. Bogucki, Tomasz Dąbrowa \\ Department of Prosthetic Dentistry, Medical University Wrocław, Poland
}

\begin{abstract}
INTRODUCTION: Tooth colour selection is one of the critical elements that guarantees a patient's satisfaction of a prosthetic restoration. There are different possible methods used in dental colour selection; however, each of them has some disadvantages.

ОвјестіVEs: The aim of the study was to compare the results of tooth colour selection carried out by means of two independent spectrophotometers using the same 3D-Master scale.

MATERIAL AND METhoDs: The material of the study included 100 prosthetic acrylic teeth by Wiedent, divided into two equal groups ( 50 each) of different colours. The colour of each tooth was examined by means of two spectrophotometers: Easyshade and SpectroShade.

RESULTs: The results of the study revealed no significant difference in hue selection between both instruments, but there were significant differences in brightness and colour intensity selection between spectrophotometers.

ConcLusions: On the basis of the study we can conclude that the instrumental method of tooth colour selection is an objective and efficient alternative to the classical visual method, but it is still not free of errors. The results of the study confirm the high utility of the spectrophotometers in tooth colour selection in dentistry, but also reveal some differences between devices from various producers. According to the results of the study, the objectivity of spectrophotometers is limited, and it is recommended that dentists use both visual and instrumental methods simultaneously..
\end{abstract}

KEY WORDS: spectrophotometer, tooth colour, shade selection.

J Stoma 2018; 71, 6: 497-501

DOI: https://doi.org/10.5114/jos.2018.85568

\section{INTRODUCTION}

The perception of colour results from the physiological reaction to the physical stimulus, which is light reflected from the surface of the observed object. Its interpretation includes both physical and physiological as well as psychological elements. Light visible to the hu- man eye is electromagnetic radiation with a wavelength between approximately 400 and $700 \mathrm{~nm}$, which is picked up by cells located in the retina of the eye, called rods and cones. Then, the physical stimulus is transformed into a nerve impulse, which is further transmitted by two separate nerve channels to the visual centre located in the cerebral cortex, where the stimulus is interpreted [1].

\section{JOURNAL OF} STOMATOLOGY CZASOPISMO STOMATOLOGICZNE

AdDress For CORRespondence: Piotr Napadłek, Department of Prosthetic Dentistry, Medical University Wrocław, 26 Krakowska St., 50-425 Wrocław, Poland, e-mail: pnapadlek@gmail.com 
Determination of the colour is therefore a complex and very subjective process. It is influenced by, among others, the following factors depending on the observer: experience, age, gender, genetic type of colour vision, eye fatigue, and emotional state. External factors that affect the perception of colour, are: intensity, temperature and angle of light rays, the colour of the dentist's surgery walls, dental unit, patient's clothes, face makeup, or background shade determined by the gums $[2,3]$.

The dynamic development of new dental materials and techniques for their use, as well as the ever-growing aesthetic requirements of patients in the world striving for perfection, pose a difficult task for a dentist in relation to the choice of colour for prosthetic restorations. The visual methods used to assess the colour of the tooth by comparison to the original colour samples can be insufficient [4]. These methods are gradually being replaced by instrumental methods, which are becoming increasingly available and popular due to their high degree of independence and precision in the choice of colour. Instrumental methods for tooth colour evaluation are colorimeters, spectrophotometers, extra- and intraoral cameras, and digital cameras. Despite considerable technological advancement, however, these devices are not without limitations and defects [5-8]. The evaluation of the real effectiveness of the spectrophotometers used and the reproducibility of the results obtained, based on their measurements, remains an unresolved issue. The null hypotheses of the study assumed uniform results for two different spectrophotometers used in colour selection of acrylic prosthetic teeth.

\section{OBJECTIVES}

The aim of the work was to research the differences between the results obtained from two independent spectrophotometers, based on the same Vita 3D-Master shade guide, used for colour selection of prosthetic teeth.

\section{MATERIAL AND METHODS}

The research was conducted in the Department of Prosthodontics of the Medical University in Wroclaw (Poland). The test material consisted of 100 acrylic upper right incisors, used in dental prosthesis, produced by the Wiedent Estetic company (Łódź, Poland). They were divided in terms of colour into two groups of 50 teeth each. The group G1 included teeth the colour of which had been designated by the producer as G1, and the group G2 included teeth that had been designated as G2. In both groups, a comparative analysis of tooth colour was carried out using two independent electronic devices for colour measurement, based on the 3D-Master scale. The evaluation of the colour of acrylic teeth on the Vita 3D-Master scale included its three components: selection of the brightness of the colour within five brightness groups, choice of colour intensity (chroma) in the three saturation groups, and selection of hue (in the scale between yellow L, middle hue $\mathrm{M}$, and red R). For its implementation, an Easyshade spectrophotometer by Vita Zahnfabrik (Bad Sackingen, Germany) and a SpectroShade by MHT SpectroShade (Verona, Italy) were used. Both devices have the option of selecting average mode values when determining the brightness and intensity of the colour.

The Easyshade spectrophotometer consists of an LCD screen, a microcomputer, and a testing tip, which contains 19 optical fibres and its own independent light source. The colour evaluation was carried out by applying a test tip, covered with a foil cover provided by the manufacturer, in the central part of the tooth's labial surface, taking into account the distance of $1 \mathrm{~mm}$ of the tip from the incisal edge and the tooth's neck. Measurements were made until three consecutive results were obtained.

The SpectroShade spectrophotometer consists of an LCD screen, a microcomputer, and a digital camera with its own independent light source. The tip with the camera covered with a casing was used to measure the colour of teeth in their middle part, until three identical results were obtained. For both devices, the teeth were placed on a black material background.

The analysis of the results was carried out using the Statistica PL version 12 computer program. The data contain quantitative and qualitative information. In the first stage of the analysis, descriptive statistics were made. To verify hypotheses regarding differences in sets of data on brightness and intensity in individual groups, Student's $t$-test for dependent variables was used. The chroma hypotheses were verified using the non-parametric Wilcoxon test.

Verification of hypotheses was made according to the following scheme:

1) checking the assumptions of the selected test (the normality of the data distribution was checked using the Shapiro-Wilk test and the homogeneity of variance using the Levene test);

2) calculation of the $p$-value by means of the chosen test (depending on the Shapiro-Wilk test result and the Levene test, parametric Student's $t$-tests for dependent variables or Wilcoxon's non-parametric tests were used);

3) deciding whether or not to reject the null hypothesis at the adopted level of significance. The significance level $\alpha=0.05$ was assumed. The $p$ values lower than 0.05 were assumed statistically significant.

\section{RESULTS}

The results of the colour brightness evaluation obtained with both spectrophotometers for the G1 group are presented in Figure 1. Analogous data for the G2 


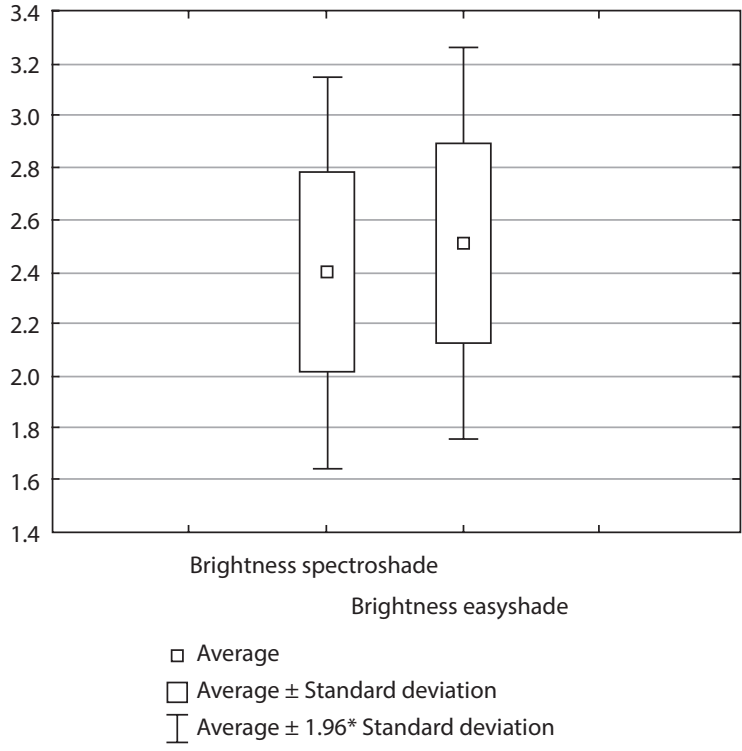

FIGURE 1. The average brightness value in the G1 group

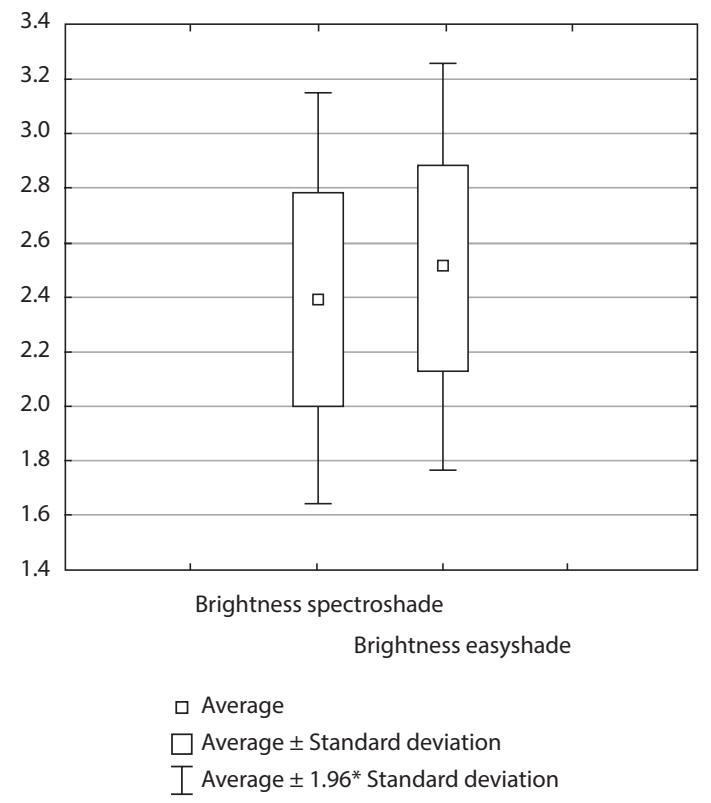

FIGURE 3. The average intensity value in the G1 group

group are presented in Figure 2. The test results showed an average brightness of 2.20 with a standard deviation of 0.29 for measurements with the Easyshade device in the G1 group, and in the G2 group average brightness of 2.93 with a standard deviation of 0.34 . For SpectroShade measurements, the average brightness in the G1 group was 2.10 with a standard deviation of 0.27 , and in the G2 group it was 2.75 with a standard deviation of 0.45 . Statistical analysis showed statistically significant differences between the measurement results from both devices.

The results of the colour intensity assessment for the G1 and G2 groups are presented in Figures 3 and 4.



FIGURE 2. The average brightness value in the $\mathrm{G} 2$ group

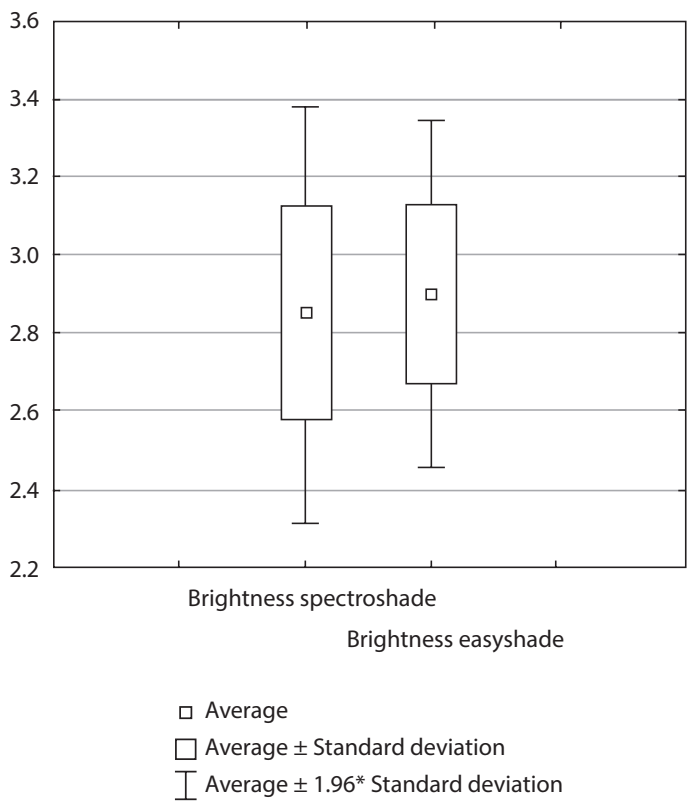

FIGURE 4. The average intensity value in the G2 group

The results of the tests showed the average intensity for Easyshade device measurements as follows: 2.51 with a standard deviation 0.38 in the G1 group, and 2.90 with a standard deviation of 0.23 in the G2 group. For SpectroShade measurements, the average intensity in the G1 group was 2.40 with a standard deviation of 0.39 , and in the G2 group it was 2.85 with a standard deviation of 0.27 . Statistical analysis showed statistically significant differences between the measurement results from both devices.

Statistical analysis did not show statistically significant differences between the measurement results of both devices for hue values. 


\section{DISCUSSION}

The choice of tooth colour is a key element during the restoration of lost tooth hard tissues as well as prosthetic reconstruction of missing teeth. It is also important to pass colour data to the prosthetic laboratory for their interpretation by a technician and finally the transfer of the data to a ready prosthetic work. The patient's final satisfaction with dental treatment is now, to an ever greater extent, determined by their satisfaction with the aesthetic result obtained, which largely depends on the correct selection of the colour of materials used in the reconstruction [9-11]. A number of studies have been conducted that compare the choice of tooth colour using conventional visual methods based on the use of shade guides, and colour selection using instrumental methods based on the use of spectrophotometers, colorimeters, digital cameras, and extra- and intraoral cameras. The results point to the imperfection of visual methods, which are subjective, because they depend on the experience of the researcher and the influence of external factors, and so they are subject to a high probability of error. On the other hand, the instrumental methods, on the basis of the results of research, are better because they are more objective, but the correctness and repeatability of the obtained results may be affected by errors resulting from improper handling or imperfection of the devices used [12-20].

The null hypotheses regarding the assessment of brightness, intensity, and hue of teeth assumed uniform results for both spectrophotometers used in both studied groups.

The results of the conducted tests showed that the average brightness measurement made with the Easyshade apparatus in the G1 group gave slightly higher values than the measurement made with the SpectroShade device. The difference was statistically significant $(p=0.0484)$. Similar differences were observed in the G2 group, in which the results of the brightness assessment obtained with the Easyshade device were higher than the results obtained using the SpectroShade device $(p=0.0066)$. The obtained results for the acrylic teeth colour intensity showed higher values for the Easyshade device than for the SpectroShade, similarly in both groups, with the statistical significance level in the G1 group, $p=0.0262$, and in the G2 group, $p=0.0580$. In the G2 group, the difference in average intensity values was "almost" statistically significant at the demarcating point with the value of $p=0.05$.

On the basis of the obtained test results, it was shown that there was no significant difference in hue assessment depending on the device used, both in the G1 group $(p=0.5165)$ and in the G2 group $(p=0.2626)$. Analysis of the obtained results of the brightness and colour intensity evaluation in the studied groups G1 and G2, with the assumed significance level $\alpha=0.05$, leads to the rejection of the null hypothesis. Analysis of the results obtained for the evaluation of acrylic teeth chroma using the Wilcoxon and Bahapkar test indicates no grounds for rejecting the verified null hypothesis. The occurrence of statistically significant differences in the scope of two of the three parameters examined between the devices suggests a certain degree of inconsistency between spectrophotometers.

The received test results, in relation to the brightness and intensity of colour, coincide with the results of Lagouvardos et al. [21], who obtained higher values for the Easyshade device compared to the ShadEye NCC spectrophotometer. Also, the results of studies by Zenthofer et al. [22], who compared the effectiveness of colour measurement with the Easyshade Advance and Easyshade Compact spectrophotometers, confirm the occurrence of discrepancies between the devices, and the existence of statistically significant difference in the accuracy of both devices $(p=0.001)$. The above observations are not confirmed by the results obtained by the Kalantari research group [23], who compared the Easyshade with the Shadepilot spectrophotometers and did not find statistically significant differences between the results. Tsiliagkou et al. [24], who compared the measurements obtained by three spectrophotometers (Easyshade, Spectroshade, and Shadevision) with regard to the Vita Classical and Vita 3D-Master shade guides (five colours from each colour palette), obtained similar results in their studies. The results obtained by the authors of the above study do not prove the lack of suitability of the spectrophotometers tested, but the necessity of a greater unification of spectrophotometers' operation abilities by the manufacturers, as well as the necessity of applying a certain amount of distrust, leading to the verification of instrumental measurement results with a visual examination using a classic shade guide [25]. The results of studies by Weyhrauch et al. [26], who compared eight copies of the Easyshade Advance spectrophotometer, indicate high reproducibility of the measurement results and their suitability in the choice of colour. The differences between our result and the results of some other authors may result from slight differences in the manner of colour measurement and the calibration of devices.

\section{CONCLUSIONS}

Based on the above research, we can conclude that there are some differences in results obtained from spectrophotometers used for colour selection. Differences in measurements between spectrophotometers suggest the need to verify the results obtained by one device, by using a different device or another method. The objectivity of spectrophotometers is limited, and it is recommended that dentists use both visual and instrumental methods simultaneously. 


\section{ACKNOWLEDGMENTS}

The authors acknowledge the assistance of the statistician Dr Andrzej Fleicher and the translator Karolina Kozakiewicz.

\section{CONFLICT OF INTEREST}

The authors declare no potential conflict of interests with respect to the authorship and/or publication of this article.

\section{References}

1. Draghici R, Preoteasa CT, Tancu AMC, Preoteasa E. Dental color assessment through TTB exercises. J Med Life 2016; 9: 61-65.

2. Gomez-Polo C, Gomez-Polo M, Martinez Vazques de Parga JA, Celemin-Vinuela A. Study of the most frequent natural tooth colors in the Spanish population using spectrophotometry. J Adv Prosthodont 2015; 7: 413-422.

3. Yamamoto S, Hosoya Y, Tsumura N, Ogawa-Ochiai K. Principal component analysis for dental shade color. Dent Mater 2012; 28 : 736-742.

4. Agarval S, Bhoyar A, Pandey SK. Shade selection in prosthodontics. Guident 2013; 3: 24-28.

5. Gomez-Polo C, Gomez-Polo M, Martinez Vazques de Parga JA, Celemin-Vinuela A. Study of natural teeth color according to toothguide 3D Master through cluster analysis. Inc Col Res Appl 2015; 41: 101-107.

6. Jgiel Ch, Lehmann KM, Ghinea R, et al. Reliability of visual and instrumental color matching. J Esthet Restor Dent 2017; 29: 303-308.

7. Sabour S, Moezizadeh M, Vahid Dastjerdi E. Reliability of shade selection using an intraoral spectrophotometer: common mistakes in reliability analysis. Clin Oral Investig 2013; 17: 1025-1031.

8. Witkowski S, Yajima ND, Wolkewitz M, Strub JR. Reliability of shade selection using an intraoral spectrophotometer. Clin Oral Investig 2012; 16: 945-949.

9. Ishikawa-Nagal S, Yoshida A, Da Silva JD, Miller L. Spectrophotometric analysis of tooth color reproduction on anterior all-ceramic crowns: part 1: analysis and interpretation of tooth color. J Comp 2010; 22: 42-52.

10. Pande N, Kolorkar MS. Spectrophotometric evaluation of shade reproduction pressable all-ceramic system on un-stained and stained tooth: an in-vitro study. J Ind Prosthodont Soc 2016; 16: 63-69.

11. Perroni AP, Bergoli CD, Bertolini M, et al. Spetrophotometric analysis of clinical factors related to the color of ceramic restorations: a pilot study. J Prosthet Dent 2017; 118: 611-616.

12. Alsaleh S, Labban M, AlHariri M, Tashkandi E. Evaluation of selfshade matching ability of dental students using visual and instrumental means. J Dent 2012; 40: 82-87.

13. Alshiddi IF, Rischards LC. A comparison of conventional visual and spectrophotometric shade taking by trained and untrained dental students. Aust Dent J 2015; 60: 176-181.

14. Brandt J, Nelson S, Lauer $\mathrm{HCH}$, et al. In vivo study for tooth colour determination-visual versus digital. Clin Oral Investig 2017; 21: 2863-2871.

15. Da Silva J, Park SE, Weber HP, Inshikawa-Nagai S. Clinical performance of a newly developed spectrophotometric system on tooth color reproduction. J Prosthet Dent 2008; 99: 361-368.

16. Gomez-Polo C, Gomez-Polo M, Celemin-Vinuela A, et al. Differences between human eye and the spectrophotometer in the shade matching of tooth colour. J Dent 2014; 42: 742-745.
17. Kroger E, Matz S, Dekiff M, et al. In vitro comparison of instrumental and visual tooth shade determination under different illuminants. J Prosthet Dent 2015; 114: 848-855.

18. Lehmann K, Devigus A, Wentaschek S, et al. Comparison of visual shade matching and electronic color measurement device. Int J Esthet Dent 2017; 12: 396-404.

19. Li Q, Wang YU, Wang YN. In vivo spectroradiometric evaluation of colour matching errors among five shade guides. J Oral Rehabil 2009; 36: 65-70.

20. Sambra Buhrer AP, Moro MG, Mazur FR, et al. Performance of dental students in shade matching: impact of training. J Esthet Restorat Dent 2017; 29: 24-32.

21. Lagouvardos PE, Fougia AG, Diamantopoulou SA, Polyzois GL. Repeatability and interdevice reliability of two portable color selection devices in matching and measuring tooth color. J Prosthet Dent 2009; 101: 40-45.

22. Zenthofer A, Cabrera T, Corcodel N, et al. Comparison of the Easyshade compact and advance in vitro and in vivo. Clin Oral Investig 2014; 18: 1473-1479.

23. Kalantari MH, Ghoraishian SA, Mohaghegh M. Evaluation of accuracy of shade selection using two spectrophotometer systems: Vita Easyshade and Degudent Shadepilot. Eur J Dent 2017; 11: 196-200.

24. Tsiliagkou A, Diamantopoulou SA, Papazoglou E, Kakaboura A. Evaluation of reliability of three dental color-matching devices. Int J Esthet Dent 2016; 11: 110-124.

25. Chu SJ, Trushkowsky RD, Paravina RD. Dental color matching instruments and systems. Review of clinical and research aspects. J Dent 2010; 38: 2-16.

26. Weyhrauch M, Igiel C, Pabst AM, et al. Interdevice agreement of eight equivalent dental color measurement devices. Clin Oral Investig 2015; 19: 2309-2318. 